\title{
(6) OPEN ACCESS \\ A systematic review and meta-analysis of studies evaluating the performance of point-of-care tests for human papillomavirus screening
}

\author{
Helen Kelly, ${ }^{1}$ Philippe Mayaud, ${ }^{1}$ Michel Segondy, ${ }^{2}$ Nitika Pant Pai, ${ }^{3}$ \\ Rosanna W Peeling ${ }^{1}$
}

\begin{abstract}
- Additional material is published online only. To view please visit the journal online (http://dx.doi.org/10.1136/ sextrans-2016-053070)

'London School of Hygiene and Tropical Medicine, London, UK INSERM U1058 and University Hospital (CHRU), Montpellier, France

${ }^{3}$ Department of Epidemiology, McGill University, Montreal, Canada
\end{abstract}

Correspondence to Dr Helen Kelly, Department of Clinical Research, Faculty of Infectious and Tropical Diseases, London School of Hygiene and Tropical Medicine, London WC1E7HT, UK; helen.kelly@ Ishtm.ac.uk

Received 7 March 2017 Revised 3 July 2017 Accepted 9 July 2017
SUMMARY

Background High-risk human papillomavirus (HPV) is a necessary cause of high-grade cervical intraepithelial neoplasia (grade 2 or higher, CIN2+). Simplified and rapid HPV DNA assays designed for use in resourcelimited settings have recently become available.

Methods We performed a systematic review and metaanalysis by searching Medline, Embase, Global Health and CINAHL databases for studies from 1 January 2004 to 25 February 2017 that reported the performance of careHPV or OncoE6 for the detection of histological CIN2+ in cervical cancer screening. We used bivariate models to estimate pooled sensitivity and specificity for $\mathrm{CIN} 2+$ and $\mathrm{CIN} 3+$

Results A total of 29657 women were included from seven studies evaluating the performance of careHPV for the detection of CIN2+ and four studies among 27845 women for the detection of CIN3+. The pooled prevalence for $\mathrm{CIN} 2+$ and $\mathrm{CIN} 3+$ was $2.3 \%$ and $1.1 \%$, respectively. careHPV had sensitivity and specificity of $88.1 \%(95 \% \mathrm{Cl} 81.4$ to 92.7$)$ and $83.7 \%(95 \% \mathrm{Cl}$ 74.9 to 89.8$)$, respectively, for CIN2+ and $90.3 \%$ (95\% $\mathrm{Cl} 83.4$ to 94.5$)$ and $85.3 \%(95 \% \mathrm{Cl} 73.1$ to 92.5$)$, respectively, for CIN3+, using clinician-collected cervical specimen. The corresponding pooled estimates using self-collected vaginal swabs were $73.6 \%$ (95\% Cl 64.9 to 80.8$)$ and $88.0 \%(95 \% \mathrm{Cl} 79.1$ to 93.5$)$ for CIN2+ and $75.2 \%(95 \% \mathrm{Cl} 66.8$ to 82.0$)$ and $90.6 \%(95 \%$ Cl 83.4 to 94.9) for CIN3+. Two studies using OncoE6 reported sensitivity and specificity ranging from $31.3 \%$ to $42.4 \%$ and $99.1 \%-99.4 \%$ for CIN2+, and $53.5 \%$ and $98.9 \%$ for CIN3+ for one study.

Conclusion CareHPV has good sensitivity and specificity for the detection of CIN2+ and CIN3+, but sensitivity was lower using self-collected vaginal samples. The specificity is lower in high HPV prevalence populations such as women living with HIV. OncoE6 assay warrants further evaluation.

\section{INTRODUCTION}

Human papillomavirus (HPV) is a necessary cause of invasive cervical cancer (ICC), ${ }^{12}$ which affects nearly 500000 women around the world every year with a mortality of more than 270000 , $80 \%$ of whom live in low and middle income (LMIC) countries. ${ }^{3}$ Cytology-based programmes have been the main approach for screening, but these are not often available in most of the low/ middle-income countries. ${ }^{4}$
The WHO 2014 cervical cancer screening guidelines recommend that screening should be performed at least once between the ages of 39 and 49 years, and this may be extended to women younger than 30 years if there is evidence of high risk for high-grade cervical intraepithelial neoplasia (grade 2 and above, CIN2+). ${ }^{56}$ However, cervical cancer screening coverage, and linkage to care, is low in LMIC countries where infrastructure and personnel requirements put high demands on the health systems. ${ }^{4}$ Cytology-based programmes have improved cervical cancer control in developed countries, but implementation in LMIC countries is constrained by cost, lack of infrastructure and trained staff, and lag time between sample collection and availability of test result, leading to delays or losses in management. ${ }^{7}$ Visual inspection of the cervix using acetic acid (VIA) or Lugol's iodine (VILI) is frequently practised in low-resource settings as it can be performed by trained midwives and nurses, and requires simple tools to perform (speculum, acetic acid, Lugol's iodine, lamp). However, frequent training and supervision is required, and the wide variation in its performance is attributed to the subjective nature of test interpretation. ${ }^{7-9}$ An important advantage of visual inspection is the possibility to link screening to immediate management.

Randomised controlled trials have shown that HPV-DNA-based screening allows for earlier diagnosis of high-grade CIN and can be more effective in prevention of ICC. ${ }^{10}$ A single round of the Digene Hybrid Capture 2 (HC2) test that detects 13 high-risk (HR) HPV types (a qualitative assay that does not distinguish the individual genotypes) has shown to halve the rate of ICC $(\mathrm{HR}=0.47,95 \% \mathrm{CI}$ 0.32 to 0.69$)$ and ICC-related mortality $(\mathrm{HR}=0.52$, $95 \% \mathrm{CI} 0.33$ to 0.83 ) compared with standardof-care among 131746 women aged 30-59 years in India. ${ }^{10}$ However, the assay is expensive and requires good logistical platforms. The addition of good-performance, rapid and affordable pointof-care (POC) tests would considerably enhance access to HPV testing in LMIC. The care HPV test (Qiagen) is based on a simplification of the $\mathrm{HC} 2$ test platform. It detects $14 \mathrm{HR}-\mathrm{HPV}$ types $(16,18$, $31,33,35,39,45,51,52,56,58,59,66$ and 68) and is optimised for low/middle-income countries: the assay needs only a small bench-top work space (about $25 \times 50 \mathrm{~cm}$ ), no mains electricity (battery operated) or running water, and can be done by 


\section{Box 1 Inclusion criteria (PICOS)}

\section{Population}

Any sexually active populations consistent with the WHO screening guidelines in any geographical location, female only, include HIV-positive patients.

\section{Interventions (index tests)}

Any commercially available technology used for HPV-POC testing in the field.

\section{Comparators}

Other commercially available technology used for HPV testing.

\section{Reference standard}

Include studies using an acceptable reference standard that satisfy the Quality Assessment of Diagnostic Accuracy Studies (QUADAS) checklist (Quality assessment-see online supplementary table 1). Acceptable reference standardhistology.

\section{Sample type}

Vaginal or cervical swab.

\section{Outcomes}

Evaluations of accuracy. Include studies calculating sensitivity, specificity, Positive Predictive Value (PPV) and Negative Predictive Value (NPV; including 95\% Cls).

\section{Study design}

Cross-sectional or cohort studies evaluating HPV-POC tests against histological endpoint of CIN2+ or CIN3+.

\section{Other}

English language only, human subjects only, 1 January 2004 to 25 February 2017.

trained staff in roughly 2.5 hours and has a longer shelf-life compared with the HC2 assay. New molecular tests with similar characteristics, although with different targets, are becoming also available on the market. The OncoE6 test (Arbor Vita) is a lateral flow immunoassay that detects the E6 oncoprotein from HPV16, HPV18 and HPV45, which cause approximately 75\% of ICC. ${ }^{11}$

The purpose of this review was to identify tests for the detection of HR-HPV that met the ASSURED criteria, ${ }^{12}$ conduct a systematic review of the literature and summarise their performance characteristics for the detection of CIN2+.

\section{METHODS}

\section{Index tests}

Only rapid/POC tests for the detection of HR HPV that met the ASSURED criteria were included (Affordable, Sensitive, Specific, User-friendly, Rapid and robust, Equipment-free and Deliverable to end-users). ${ }^{12}$ HR-HPV types were defined using the current International Agency for Research on Cancer classification ${ }^{13}$ : 'carcinogenic to humans' (HPV16, 18, 31, 33, 35, 39, 45, 51, $52,56,58,59)$ and 'probable carcinogenic' (HPV68).

\section{Reference or gold standard test}

Studies were included if HPV-DNA tests were assessed against a histological endpoint of CIN grade 2 or higher $(\mathrm{CIN} 2+)$. Studies with cytological endpoint assessment only were excluded because of the lower sensitivity for cytology measures in detection of high-grade disease. ${ }^{8}$

\section{Search}

We searched Medline, Embase, Global Health and CINAHL databases for publications in the English language from 1 January 2004 to 31 December 2013 using search terms for human papillomavirus (HPV), cervical intraepithelial lesions (CIN), squamous intraepithelial lesions (SIL), HPV screening; Diagnostics; Point of Care; Rapid; Near patient; method; test; evaluation; performance and comparison. Reference lists of review articles and all articles identified in the systematic search were checked. An updated search was performed on 25 February 2017. All abstracts were screened by one author (HK).

\section{Inclusion and exclusion criteria}

Eligibility criteria were defined using the PICOS (Population, Interventions, Comparisons, Outcomes, Study Design) criteria as shown in box 1. Studies evaluating the accuracy of any HPV-POC tests commercially available at the time of the review were considered for inclusion.

Studies were excluded if the index test was not a rapid test for HPV (ie, did not meet the ASSURED criteria), if diagnostic accuracies were not compared with a histological endpoint and if the studies did not report data to allow for calculation of diagnostic accuracy.

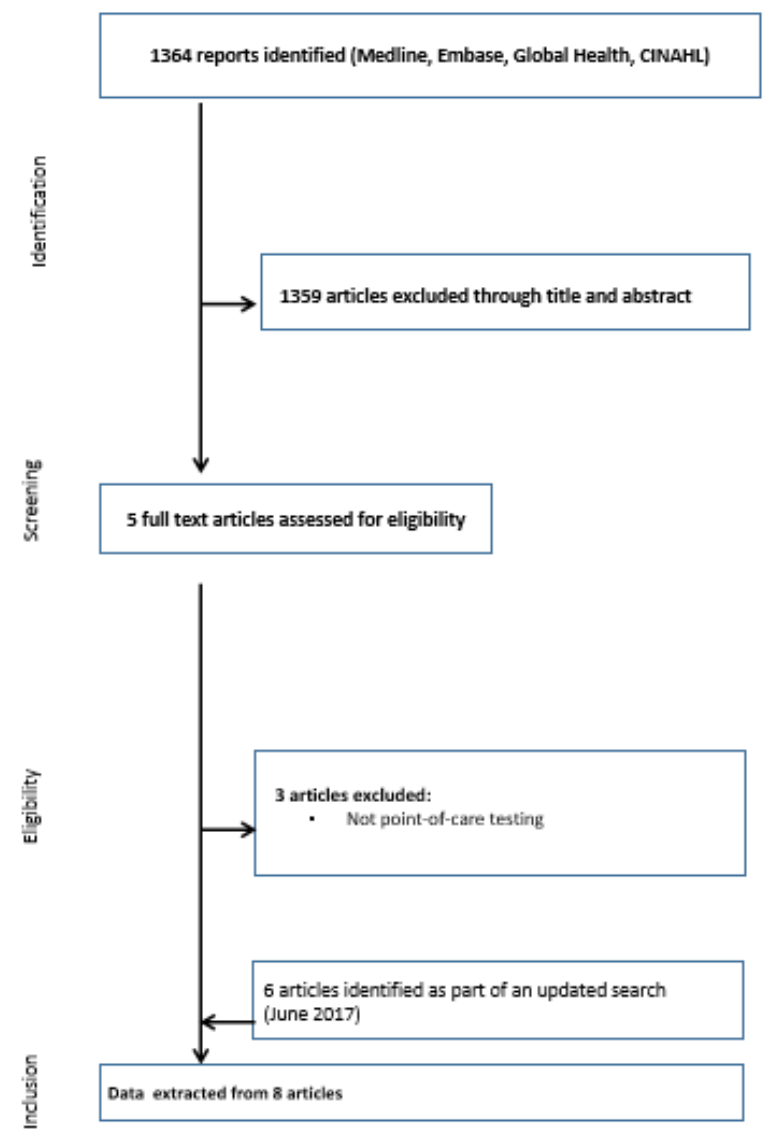

Figure 1 Flowchart for study selection. 


\section{Data extraction}

From the consensus list, sensitivity and specificity measures were extracted by one author (HK) using a standardised form. For all studies, the following variables were recorded: year of study, study location, study population, outcomes of interest (histological confirmed lesion CIN2+/CIN3+), HR-HPV prevalence and sensitivity and specificity for the detection of CIN2+ and $\mathrm{CIN} 3+$, where given.

\section{Methodological assessment}

Included studies were assessed according to the QUADAS-2 checklist for reporting diagnostic accuracy ${ }^{14}$ (online supplementary table 1).

\section{Statistical analysis}

The numbers of true positives, false positives, true negatives and false negatives were extracted where available. Where sensitivity and specificity measures only were given, data were extracted to calculate the numbers of true positives, false negatives, true negatives and false positives. Forest plots were generated to display sensitivity and specificity estimates.

\section{Meta-analysis}

The bivariate model ${ }^{15}$ was used to estimate pooled sensitivity and specificity estimates using metandi and midas in STATA, whereby pairs of sensitivity and specificity are jointly analysed, incorporating any correlation that might exist between these two measures using a random-effects approach. Heterogeneity across studies was assessed using the $\mathrm{I}^{2}$ statistic. ${ }^{16}$

Separate meta-analyses were performed for the detection of CIN2 + and CIN3 + outcomes, and were also performed separately for studies that used different specimen types (cervical vs vaginal swabs).

To account for correlation between sensitivity and specificity, we used the hierarchical summary receiver operating characteristic (HSROC), ${ }^{17}$ which allows for threshold effects and between-study and within-study variability, by allowing both test accuracy to vary across studies. The heterogeneity in the forest plots were assessed by visually examining the confidence intervals (CIs) of individual studies, and in summary HSROC plots by examining the width of the prediction region, with a wider prediction region suggesting more heterogeneity.

\section{Sensitivity analysis}

Sensitivity analyses could not be performed due to lack of additional covariate data. Formal assessment of publication bias was not performed due to low number of studies.

Data were analysed using Stata V.14 (Stata Statistical Software).

This review was reported according to the Preferred Reporting Items for Systematic Reviews and Meta-Analyses (PRISMA) ${ }^{18}$ and the Meta-analysis of Observational Studies in Epidemiology (MOOSE) guidelines. ${ }^{19}$

\section{RESULTS}

\section{Search result}

The first review up to 31 December 2013 identified 1364 publications through Medline, Embase, Global Health and CINAHL library searches, which reported performance measures for HPV DNA point-of-care tests for the detection of CIN2+ (figure 1), of which 1359 were excluded after abstract review because the assays did not meet the ASSURED criteria, leaving five publications for full-text review. Finally, two publications ${ }^{2021}$ satisfied the inclusion criteria. An updated search performed until
25 February 2017 identified an additional four publications ${ }^{22-25}$ that satisfied the inclusion criteria. Two further publications ${ }^{26} 27$ were subsequently identified in June 2017. The reviews identified only two tests that appeared to satisfy the ASSURED criteria (careHPV (Qiagen) targeting the HR-HPV types HPV16/18/31 /33/35/39/45/51/52/56/58/59/66/68 and OncoE6 (Arbor Vita) targeting the HR-HPV types HPV16/18/45), which had publication material that could be assessed. The characteristics of the included publications are summarised in Table 1. One publication $^{24}$ provided performance estimates for two countries and was considered as two separate populations in the analysis; one publication ${ }^{23}$ provided performance estimates for four countries and was considered as four separate populations in the analysis, and one publication ${ }^{27}$ provided performance estimates separately for women living with HIV (WLHIV) and HIV-negative women and was considered as two populations. This resulted in 12 separate populations included in the final analysis, among 29657 women. Two studies measured the performance of care HPV for CIN2 + detection among 1215 WLHIV in Burkina Faso and South Africa, ${ }^{24}$ and Uganda. ${ }^{27}$

Among seven studies (twelve populations) evaluating the careHPV test (table 1), all evaluated the performance of care HPV test for the detection of $\mathrm{CIN} 2+$ and four studies (eight populations) evaluated its performance for the detection of CIN3 $+{ }^{2022-24}$ using clinician-collected cervical swabs. Four studies (eight populations) evaluated the performance of careHPV for the detection of CIN2 $+{ }^{20222327}$ and three studies (six populations) for CIN3 $+{ }^{2022} 23$ using self-sampled vaginal swabs. All studies evaluating the performance of careHPV used a cut-off of $1 \mathrm{pg} / \mathrm{mL}$ for defining test positivity.

Two studies evaluated the performance of OncoE6 test for the detection of CIN $2+{ }^{22} 25$ and one study for CIN $3+{ }^{22}$ using clinician-collected cervical swabs.

\section{The CareHPV test for the detection of CIN2+ and CIN3+}

The sensitivity and specificity estimates for CIN2+ for individual populations are summarised in figure 2 (clinician-collected cervical swabs) and figure 3 (self-collected vaginal swabs). Sensitivity and specificity for CIN3 + for individual populations are summarised in figure 4 (clinician-collected cervical swab) and figure 5 (self-collected vaginal swab). The pooled summary estimates are summarised in table 2.

The careHPV test had a sensitivity of $88.1 \%$ (95\% CI 81.4 to 92.7) and specificity of $83.7 \%$ (95\% CI 74.9 to 89.8 ) for CIN2+ detection using clinician-collected cervical swabs (table 2), and a sensitivity of $73.6 \%$ (95\% CI 64.9 to 80.8 ) and specificity of $88.0 \%$ (95\% CI 79.1 to 93.5 ) using self-collected vaginal swabs.

The corresponding pooled estimates for sensitivity and specificity for CIN3 + were 90.3\% (95\% CI 83.4 to 94.5$)$ and $85.3 \%$ (95\% CI 73.1 to 92.5 ), respectively, using cervical swabs and $75.2 \%$ (95\% CI 66.8 to 82.0 ) and $90.6 \%$ (95\% CI 83.4 to 94.9 ) using vaginal swabs. Heterogeneity across studies was high for both CIN2 + and CIN3 + outcomes (table 2).

When restricting the analysis to six studies (nine populations) among the general population of women using clinician-collected cervical swabs, the sensitivity for CIN2+ was similar to the overall pooled result $(84.9 \%, 95 \%$ CI 76.8 to 90.5 ; table 2$)$, but specificity was higher $(88.6 \%, 95 \%$ CI 76.8 to 90.5$)$. The pooled estimates for the 1215 WLHIV in Burkina Faso, South Africa and Uganda were 93.7\% (95\% CI 86.8 to 97.7) and $58.8 \%$ (95\% CI 55.9 to 61.7 ) for sensitivity and specificity, respectively (data not shown). ${ }^{24} 27$ 


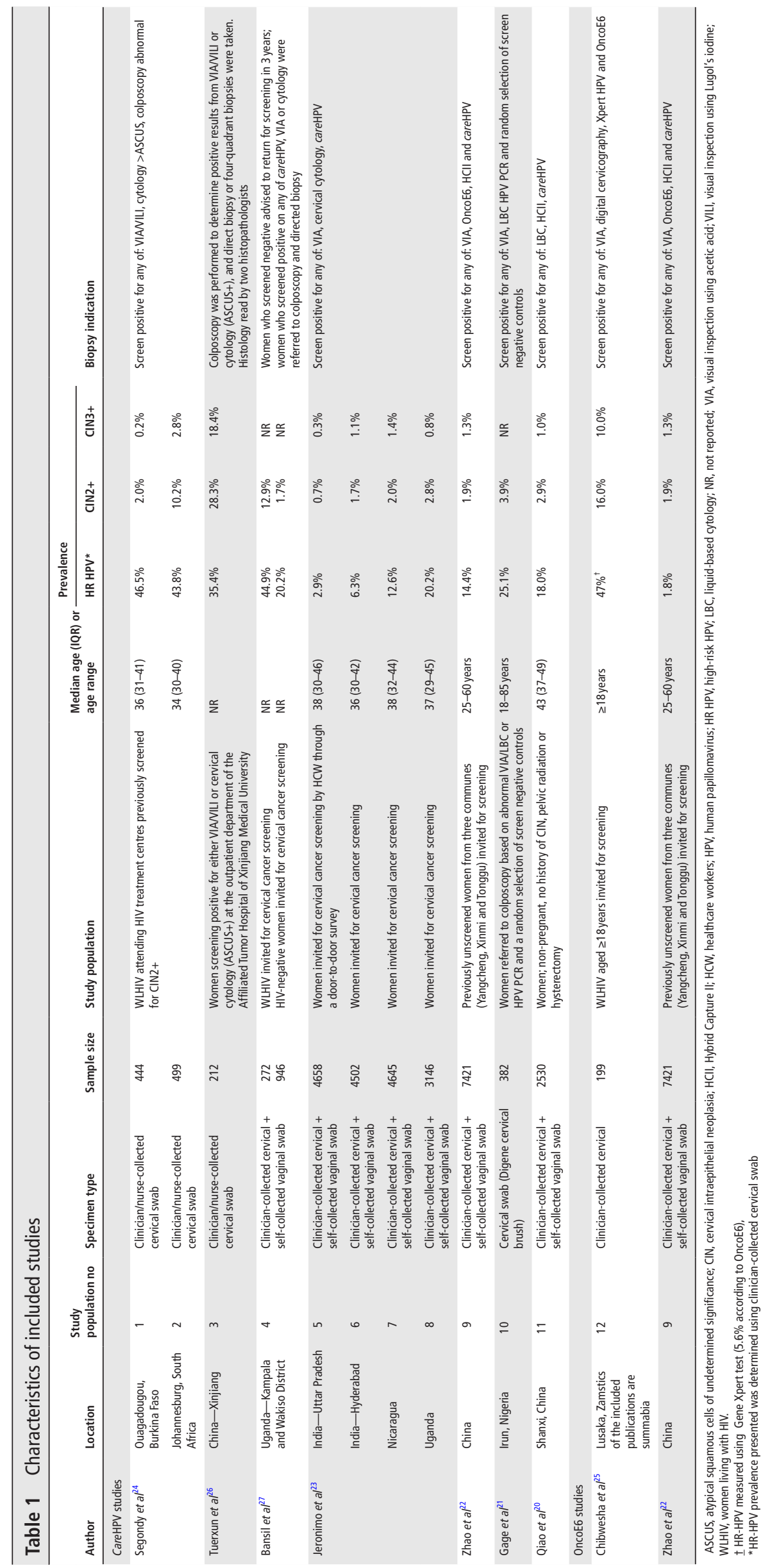




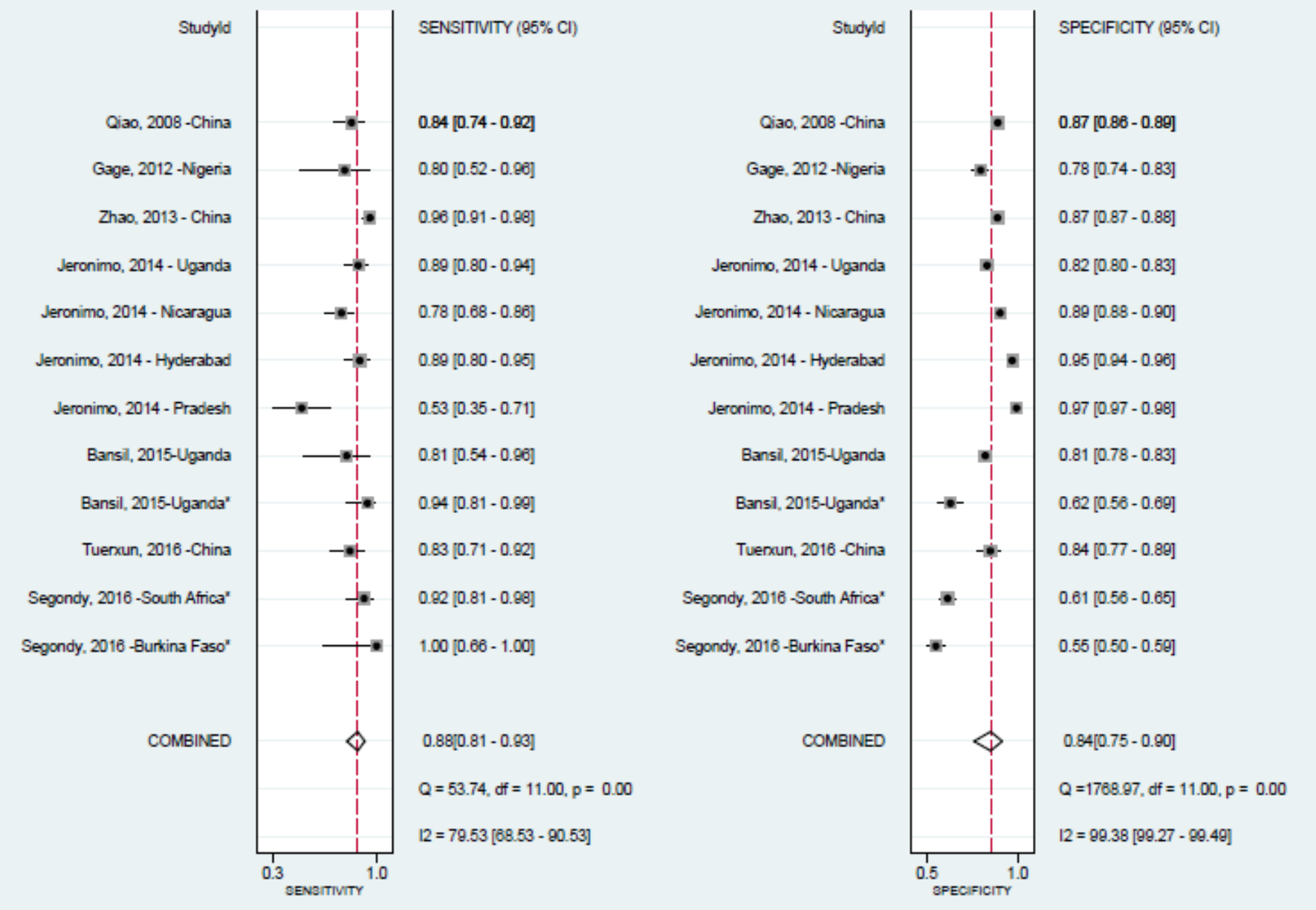

Figure 2 Meta-analysis of the careHPV test performance (sensitivity and specificity) for CIN2+ detection using clinician-collected cervical swabs among 12 populations. * studies among women living with HIV

OncoE6 for the detection of CIN2+ and CIN3+

There were two studies that evaluated the performance of OncoE6 for the detection of CIN2 $+{ }^{22} 25$ among 7621 women and CIN3+ among 7421 women, ${ }^{22}$ and among 200 WLHIV. ${ }^{25}$ Zhao et $\mathrm{al}^{22}$ reported a sensitivity and specificity of $42.4 \%$ and $99.1 \%$, respectively, for the detection of CIN2+, and $53.5 \%$ and $98.9 \%$, respectively, for the detection of CIN3 + among HIV-negative women in China. In Zambia, Chibwesha et al ${ }^{25}$ reported a sensitivity and specificity of $31.3 \%$ (95\% CI 16 to 50 ) and $99.4 \%$ (95\% CI 97 to 100), respectively, for CIN2+ detection among WLHIV.

\section{Methodological assessment}

According to the QUADAS criteria (online supplementary table 1 ), the quality of the papers included was acceptable, but with some omissions in reporting on the training of test operators, mechanism for blinding of index and reference test and how indeterminate results were handled.

\section{DISCUSSION}

This review found that the careHPV test had good sensitivity and specificity for the detection of CIN2+ $(88.1 \%$ and $83.7 \%$, respectively) and CIN3 + (90.3\% and $85.3 \%$, respectively) using clinician-collected swabs. While specificity remained high when using self-collected vaginal swabs, the sensitivity for CIN2 + and CIN3 + was lower than when using clinician-collected swabs (73.6\% and $75.2 \%$, respectively).

The pooled performance estimates in this review suggest that while the care HPV test has similar specificity to that reported for the Hybrid Capture II (HCII) assay, the sensitivity is lower than what has been previously reported in other meta-analyses of HCII performance. ${ }^{28}$ This is consistent with a recent meta-anal$\mathrm{ysis}^{29}$ that directly compared the performance of the two assays and reported a relative sensitivity for the care HPV test compared with the HCII assay of $0.86(95 \%$ CI 0.79 to 0.94$)$ and relative specificity of 1.01 (95\% CI 0.99 to 1.03 ) for CIN2+.

Compared with the other screening modalities, visual inspection and cervical cytology, the careHPV test had higher sensitivity but similar specificity for the detection of CIN2+. In a meta-analysis of 21 studies from sub-Saharan Africa and India among over 58000 women, ${ }^{78}$ VIA showed moderate sensitivity for the detection of CIN2+ (pooled estimate: 79.2\%-82.4\%) and specificity $(84.7 \%-87.4 \%)$. VILI performed even better with higher sensitivity (CIN2+: 91.2\%-95.1\%; CIN3+: 93.8\%) and similar specificity (CIN2+: $84.5 \%-87.2 \%$; CIN3 + : $83.8 \%)$. However, sensitivity has been reported to be as low as $50 \%$ in other studies. ${ }^{9}$ The wide variation in its performance is attributed to the subjective nature of test interpretation. High 


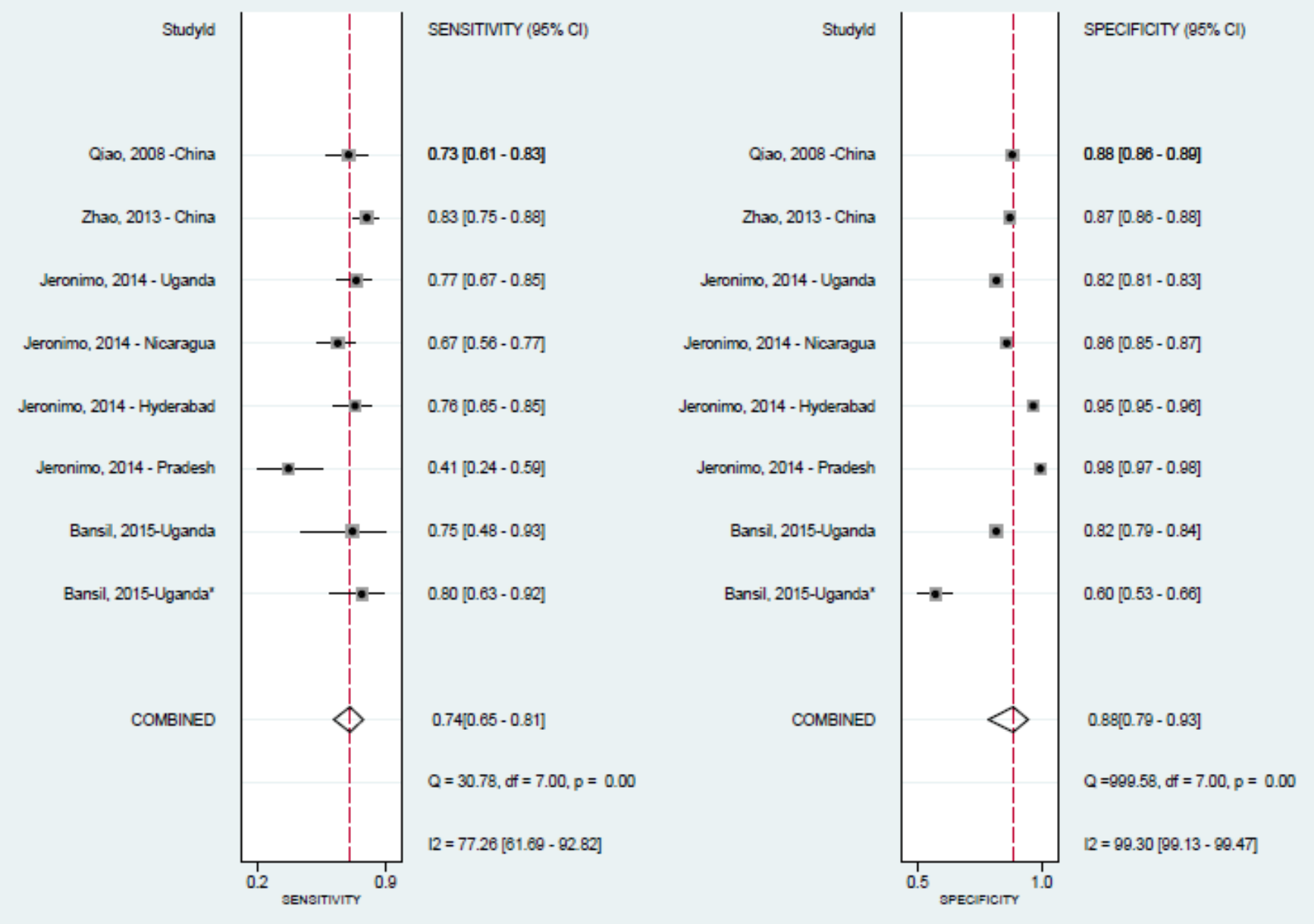

Figure 3 Meta-analysis of the careHPV test performance (sensitivity and specificity) for CIN2+ detection using self-collected vaginal swabs among eight populations. *studies among women living with HIV

rates of positivity have resulted in over-referral to colposcopy services and overtreatment. ${ }^{30}$ Despite this, a single round of VIA screening has been associated with a $25 \%-35 \%$ reduction in cervical cancer incidence, cervical cancer mortality and the frequency of CIN2 + lesions, in studies in Thailand, Ghana and Zambia. ${ }^{31-33}$

The careHPV test also performed better than cervical cytology. In a meta-analysis of studies evaluating performance of screening in Africa and India, ${ }^{8}$ cytology, whether using positive test result cut-offs of atypical squamous cells of undetermined significance and greater ( $\geq$ ASCUS), low-grade squamous intraepithelial lesions ( $\geq$ LSIL) or high-grade squamous intraepithelial lesions ( $\geq$ HSIL), had poor sensitivity for the detection of CIN2+ (range: 42.6 for $\geq$ HSIL to $57.0 \%$ for $\geq$ ASCUS) and CIN3 + (range: 51.6\%-63.0\%) but high specificity (range: 92.8\%-99.3\% and 92.3\%-99.0\% for CIN2+ and CIN3 +, respectively).

\section{HPV-DNA testing among women living with HIV}

The careHPV test was shown to have good sensitivity but lower specificity for the detection of CIN2+ among WLHIV in Burkina Faso, South Africa and Uganda in this review compared with the general population, ${ }^{24} 27$ similar to what was reported for HCII among WLHIV in South Africa. ${ }^{34}$ HPV testing may detect many transient infections, which do not progress to CIN2+, meaning that its specificity for high-grade CIN can be low. A recent review of HPV-DNA-based tests for the detection of cervical lesions reported that for a $10 \%$ increase in HR-HPV prevalence, HR-HPV-DNA-based test specificity for CIN2+ detection decreased by $8.4 \%$ (95\% CI $8.0 \%$ to $8.8 \%),{ }^{35}$ which has important implications for HIV-positive populations who have a high prevalence of HR HPV. Segondy et al also reported an increase in specificity with increasing CD4+ cell count and among women on antiretroviral therapy (ART), corresponding with a decrease in HR-HPV prevalence with increasing CD4+ cell count and prolonged duration of ART use. Studies have shown that women are at decreased risk of CIN2 + if ART is initiated at higher CD $4+$ counts $\left(>500\right.$ cells $\left./ \mathrm{mm}^{3}\right),{ }^{36}$ and prolonged ART duration and high CD4+ cell count are associated with a reduction in HR-HPV prevalence and persistence. ${ }^{37} 38$

The biggest contribution of POC-HPV testing is the possibility of decentralising screening, increasing the number of women who enter the screening and management process more promptly and therefore may ultimately contribute to the decrease of incidence of high-grade cervical lesions. The slightly lower sensitivity and specificity for vaginal self-sampling compared with clinician-collected cervical samples in this review were similar to that in a large meta-analysis that 


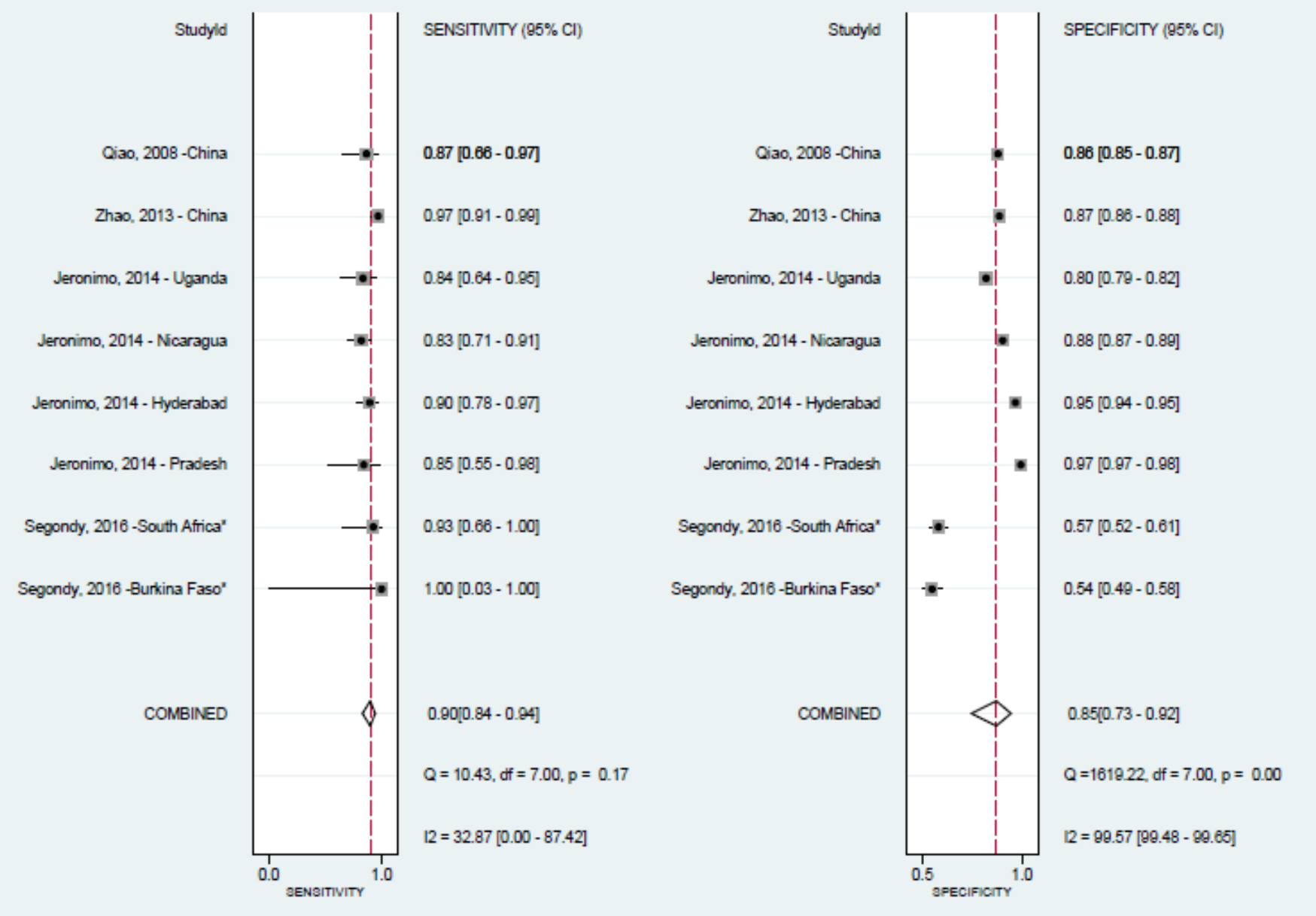

Figure 4 Meta-analysis of the careHPV test performance (sensitivity and specificity) for CIN3+ detection using clinician-collected cervical swabs among eight populations. *studies among women living with HIV

reported a relative sensitivity of 0.88 (95\% CI 0.85 to 0.91$)$ and specificity of 0.96 ( $95 \%$ CI 0.95 to 0.97 ) for CIN2+ using vaginal self-sampling compared with clinician-collected cervical samples. ${ }^{39}$ However, the possibility of self-sampling may allow for an increase in uptake of testing especially in settings where self-sampling might be preferred for cultural or logistical reasons. A number of studies have evaluated the acceptability and feasibility of the self-sampling using the care HPV test, and for some the concordance of results. A study of 431 women in rural Roi-et Province in Thailand ${ }^{40}$ reported that $91 \%$ found the self-swabbing acceptable, $71 \%$ preferred the self-swab to a clinician swab and $95 \%$ returned for same-day follow-up. In a study among 518 women in El Salvador, ${ }^{41}$ $38.8 \%$ preferred self-collection and $31.9 \%$ preferred provider collection. In a study among 194 women in Ghana $(50 \%$ of whom are WLHIV), 76.3\% women found self-collection very easy/easy to obtain, $57.7 \%$ preferred self-collection to clinician collection and $61.9 \%$ felt self-collection would increase their likelihood to access cervical cancer screening; in that study, the concordance of the careHPV test results between self-collected and clinician-collected samples was $94.2 \%{ }^{42}$ Hence, despite the lower sensitivity of the careHPV test using vaginal self-swabs in this review, self-collection coupled with POC testing has the potential to increase coverage of screening and impact on disease rates. Prospective studies are warranted to compare the effectiveness of vaginal self-sampling over clinic-based cervical sampling in terms of numbers of CIN identified and managed.

The low sensitivity but high specificity of the OncoE6 test, which targets HPV16, HPV18 and HPV45, for CIN2+ detection suggests it may be useful as a 'screen-and-treat' or triage test; however, further studies are warranted, especially in settings where HPV vaccination has been introduced.

\section{Limitations}

A major drawback of the evaluation of HPV POC tests against a relatively rare disease outcome such as CIN2+ is that it is not a direct comparison of the detection of the infection that may cause disease several years later. Most HR-HPV infections are transient and will not all cause severe histological changes. Therefore, a measure of persistent infection at two time points, or a marker of transformative or integrative infection, might increase specificity. Furthermore, it is believed that some HR-HPV types, such as HPV16, HPV18 and HPV45, might have greater oncogenic potential.

There was significant heterogeneity across studies $\left(\mathrm{I}^{2}\right.$, table 2), which may be attributed to the variation in HR-HPV 


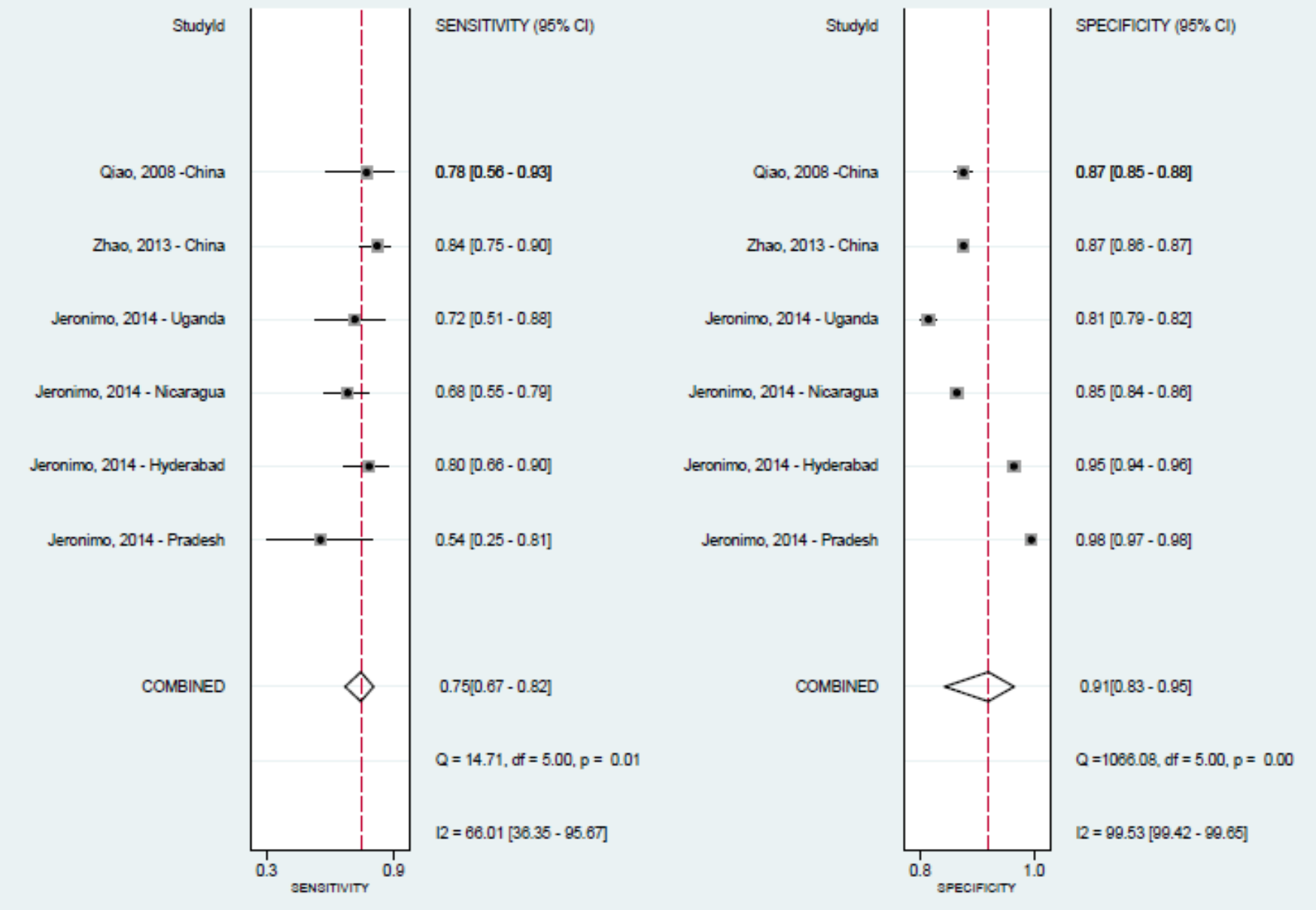

Figure 5 Meta-analysis of the careHPV test performance (sensitivity and specificity) for CIN3+ detection using self-collected vaginal swabs among six populations.

prevalence across studies. WLHIV in Burkina Faso, South Africa and Uganda had the highest prevalence (46.5\%, 43.8\% and $44.9 \%$, respectively), but excluding these studies did not lead to a reduction in heterogeneity. HR HPV was also high among HIV-negative women in Uganda (20.2\%), Nigeria $(25.1 \%)$ and China $(18.0 \%)$. Another study in Rwanda ${ }^{43}$ reported that the careHPV test positivity was higher among 1289 WLHIV compared with 1675 HIV-negative women ( $31.8 \%$ vs $8.2 \%$, respectively), but the study did not measure its performance for $\mathrm{CIN} 2+$ detection.

There was potential bias attributed to biopsy indication in the included studies. Most studies maximised the chances of obtaining histological results by basing biopsy decision on positivity of any of the screening tests, which included visual inspection, cervical cytology and HPV-DNA tests. Women negative by all tests were considered to be at extremely low risk of CIN2 since in particular HPV DNA and cytology have very high negative predictive values for CIN2 diagnosis. ${ }^{44}$ There is potentially greater risk of ascertainment bias in studies with lower prevalence of HR HPV (table 2), especially among the studies that used self-collected vaginal specimens which had lower sensitivity for CIN2+. Relative accuracy estimates of the careHPV test compared with other HPV assays would allow for a comparability measure of the different HPV assays, taking into account the heterogeneity across studies and the effects of sampling devices, quality and histological endpoint determination. There were too few studies evaluating the performance of the OncoE6 test to conduct a formal pooling analysis.

New tests for HPV detection, such as the Xpert HPV, look promising in terms of performance for the detection of CIN2+ compared with HC2 or HPV genotyping, ${ }^{25} 4546$ but were not considered to satisfy the ASSURED criteria for this review. Over time and with lower costs, such tests may qualify, especially if investment is made in the platform, as is the case for countries like South Africa, which has introduced Xpert for Mycobacterium tuberculosis diagnosis nationwide.

\section{CONCLUSION}

The careHPV test performs as well as other screening modalities, such as visual inspection and cervical cytology, and has the added advantage of allowing for self-sampling that can increase coverage and uptake of screening. HPV-DNA testing is, however, less specific among WLHIV and could result in more false-positive results and over-referral for colposcopy in this population. The possibility for future HPV-POC tests to distinguish between certain HPV types, to determine persistence or transformation/integration stages, might improve on their performance. 


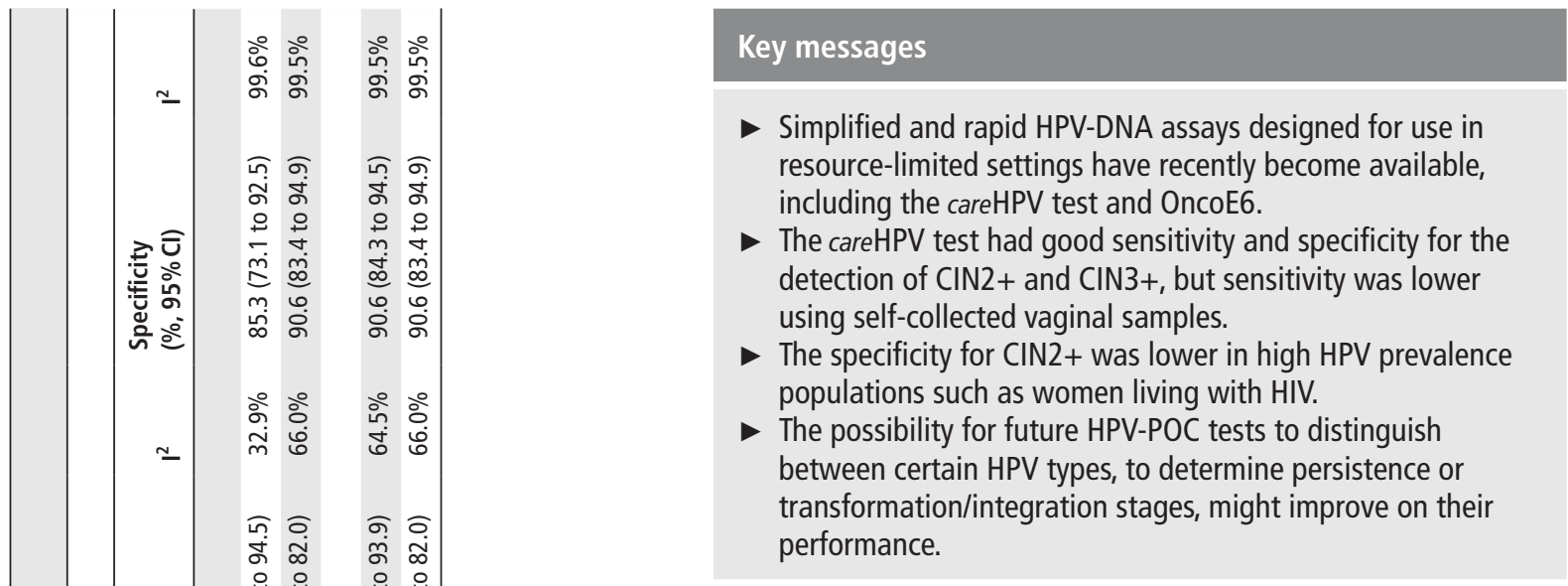

Handling editor Jackie A Cassell

Contributors HK, PM and RWP conceptualised the study and developed the research protocol. HK and PM identified articles for full-text review. HK extracted data from studies that matched inclusion criteria. HK and NPP did the statistical analyses. All contributed to the writing of the manuscript.

Funding The authors of this manuscript received funding support from the UK Medical Research Council (MRC) PHINDS scheme (PH01/14-39).

Competing interests None declared.

Provenance and peer review Commissioned; externally peer reviewed.

Open Access This is an Open Access article distributed in accordance with the terms of the Creative Commons Attribution (CC BY 4.0) license, which permits others to distribute, remix, adapt and build upon this work, for commercial use, provided the original work is properly cited. See: http://creativecommons.org/licenses/by/4.0/

(c) Article author(s) (or their employer(s) unless otherwise stated in the text of the article) 2017. All rights reserved. No commercial use is permitted unless otherwise expressly granted.

\section{REFERENCES}

1 Bosch FX, Lorincz A, Muñoz N, et al. The causal relation between human papillomavirus and cervical cancer. J Clin Pathol 2002;55:244-65.

2 Walboomers JM, Jacobs MV, Manos MM, et al. Human papillomavirus is a necessary cause of invasive cervical cancer worldwide. J Pathol 1999;189:12-19.

3 Globocan. Estimated cancer incidence, mortality and prevalence worldwide in 2012. http://globocan.iarc.fr (accessed 31 Dec 2016).

4 Denny L, Quinn M, Sankaranarayanan R. Chapter 8: screening for cervical cancer in developing countries. Vaccine 2006;24(Suppl 3):S71-77.

5 WHO. Comprehensive cervical cancer control: a guide to essential practice. 2nd edition, 2014. (accessed 12 Jan 2017).

6 Santesso N, Mustafa RA, Schünemann HJ, et al. World Health Organization Guidelines for treatment of cervical intraepithelial neoplasia 2-3 and screen-and-treat strategies to prevent cervical cancer. Int J Gynaecol Obstet 2016;132:252-8.

7 Fokom-Domgue J, Combescure C, Fokom-Defo V, et al. Performance of alternative strategies for primary cervical cancer screening in sub-Saharan Africa: systematic review and meta-analysis of diagnostic test accuracy studies. BMJ 2015;351:h3084.

8 Arbyn M, Sankaranarayanan R, Muwonge R, et al. Pooled analysis of the accuracy of five cervical cancer screening tests assessed in eleven studies in Africa and India. Int J Cancer 2008;123:153-60.

9 Sankaranarayanan R, Nessa A, Esmy PO, et al. Visual inspection methods for cervical cancer prevention. Best Pract Res Clin Obstet Gynaecol 2012;26:221-32.

10 Sankaranarayanan R, Nene BM, Shastri SS, et al. HPV screening for cervical cancer in rural India. N Engl J Med 2009;360:1385-94.

11 de Sanjose S, Quint WG, Alemany L, et al. Human papillomavirus genotype attribution in invasive cervical cancer: a retrospective cross-sectional worldwide study. Lancet Oncol 2010;11:1048-56.

12 Peeling RW, Holmes KK, Mabey D, et al. Rapid tests for sexually transmitted infections (STIs): the way forward. Sex Transm Infect 2006;82(Suppl 5):v1-6.

13 Bouvard V, Baan R, Straif K, et al. On behalf of the WHO International Agency for Research on Cancer Monograph Working Group. A review of human carcinogenspart B: biological agents. Lancet Oncology 2009;10:321-2.

14 Whiting PF, Rutjes AW, Westwood ME, et al. QUADAS-2: a revised tool for the quality assessment of diagnostic accuracy studies. Ann Intern Med 2011;155:529-36.

15 Reitsma JB, Glas AS, Rutjes AW, et al. Bivariate analysis of sensitivity and specificity produces informative summary measures in diagnostic reviews. J Clin Epidemiol 2005;58:982-90. 
16 Higgins JP, Thompson SG. Quantifying heterogeneity in a meta-analysis. Stat Med 2002;21:1539-58.

17 Rutter CM, Gatsonis CA. A hierarchical regression approach to meta-analysis of diagnostic test accuracy evaluations. Stat Med 2001;20:2865-84.

18 Moher D, Liberati A, Tetzlaff J, et al. Preferred reporting items for systematic reviews and meta-analyses: the PRISMA statement. BMJ 2009:339:b2535.

19 Stroup DF, Berlin JA, Morton SC, et al. Meta-analysis of observational studies in epidemiology: a proposal for reporting. Meta-analysis Of Observational Studies in Epidemiology (MOOSE) group. JAMA 2000;283:2008-12.

20 Qiao YL, Sellors JW, Eder PS, et al. A new HPV-DNA test for cervical-cancer screening in developing regions: a cross-sectional study of clinical accuracy in rural China. Lancet Oncol 2008;9:929-36.

21 Gage JC, Ajenifuja KO, Wentzensen N, et al. Effectiveness of a simple rapid human papillomavirus DNA test in rural Nigeria. Int J Cancer 2012;131:2903-9.

22 Zhao FH, Jeronimo J, Qiao YL, et al. An evaluation of novel, lower-cost molecular screening tests for human papillomavirus in rural China. Cancer Prev Res 2013;6:938-48.

23 Jeronimo J, Bansil P, Lim J, et al. A multicountry evaluation of careHPV testing, visual inspection with acetic acid, and Papanicolaou testing for the detection of cervical cancer. Int J Gynecol Cancer 2014;24:576-85.

24 Segondy M, Kelly H, Magooa MP, et al. Performance of careHPV for detecting highgrade cervical intraepithelial neoplasia among women living with HIV-1 in Burkina Faso and South Africa: HARP study. Br J Cancer 2016;115:425-30.

25 Chibwesha CJ, Frett B, Katundu K, et al. Clinical performance validation of 4 pointof-care cervical cancer screening tests in HIV-infected women in Zambia. J Low Genit Tract Dis 2016;20:218-23.

26 Tuerxun G, Yukesaier A, Lu L, et al. Evaluation of careHPV, Cervista Human Papillomavirus, and Hybrid Capture 2 methods in diagnosing cervical intraepithelial neoplasia grade 2+ in Xinjiang Uyghur women. Oncologist 2016;21:825-31.

27 Bansil P, Lim J, Byamugisha J, et al. Performance of cervical cancer screening techniques in HIV-infected women in Uganda. J Low Genit Tract Dis 2015;19:215-9.

28 Arbyn M, Ronco G, Anttila A, et al. Evidence regarding human papillomavirus testing in secondary prevention of cervical cancer. Vaccine 2012;30(Suppl 5):F88-99.

29 Arbyn M, Snijders PJ, Meijer CJ, et al. Which high-risk HPV assays fulfil criteria for use in primary cervical cancer screening? Clin Microbiol Infect 2015:21:817-26.

30 Denny L, de Sanjose S, Mutebi M, et al. Interventions to close the divide for women with breast and cervical cancer between low-income and middle-income countries and high-income countries. Lancet 2017;389.

31 Gaffikin L, Blumenthal PD, Emerson M, et al. Safety, acceptability, and feasibility of a single-visit approach to cervical-cancer prevention in rural Thailand: a demonstration project. Lancet 2003:361:814-20.

32 Blumenthal PD, Gaffikin L, Deganus S, et al. Cervical cancer prevention: safety, acceptability, and feasibility of a single-visit approach in Accra, Ghana. Am J Obstet Gynecol 2007;196:407.e1-9.
33 Parham GP, Mwanahamuntu MH, Kapambwe S, et al. Population-level scale-up of cervical cancer prevention services in a low-resource setting: development, implementation, and evaluation of the cervical cancer prevention program in Zambia. PLoS One 2015;10:e0122169.

34 Firnhaber C, Mayisela N, Mao L, et al. Validation of cervical cancer screening methods in HIV positive women from Johannesburg South Africa. PLoS One 2013;8:e53494.

35 Giorgi-Rossi P, Franceschi S, Ronco G. HPV prevalence and accuracy of HPV testing to detect high-grade cervical intraepithelial neoplasia. Int J Cancer 2012;130:1387-94.

36 Huchko MJ, Leslie H, Sneden J, et al. Risk factors for cervical precancer detection among previously unscreened HIV-infected women in Western Kenya. Int J Cancer 2014;134:740-5

37 Kelly HA, Sawadogo B, Chikandiwa A, et al. Epidemiology of high-risk human papillomavirus and cervical lesions in african women living with HIVIAIDS: effect of anti-retroviral therapy. AIDS 2016.

38 Konopnicki D, Manigart Y, Gilles C, et al. Sustained viral suppression and higher CD4+ T-cell count reduces the risk of persistent cervical high-risk human papillomavirus infection in HIV-positive women. J Infect Dis 2013:207:1723-9.

39 Arbyn M, Verdoodt F, Snijders PJ, et al. Accuracy of human papillomavirus testing on self-collected versus clinician-collected samples: a meta-analysis. Lancet Oncol 2014; 15:172-83.

40 Trope LA, Chumworathayi B, Blumenthal PD. Feasibility of community-based careHPV for cervical cancer prevention in rural Thailand. J Low Genit Tract Dis 2013;17:315-9.

41 Rosenbaum AJ, Gage JC, Alfaro KM, et al. Acceptability of self-collected versus provider-collected sampling for HPV DNA testing among women in rural El Salvador. Int J Gynaecol Obstet 2014;126:156-60.

42 Obiri-Yeboah D, Adu-Sarkodie Y, Djigma F, et al. Self-collected vaginal sampling for the detection of genital human papillomavirus (HPV) using careHPV among Ghanaian women. BMC Women's Health 2017.

43 Sinayobye J, Sklar M, Hoover DR, et al. Prevalence and risk factors for High-Risk Human Papillomavirus (hrHPV) infection among HIV-infected and uninfected Rwandan women: implications for hrHPV-based screening in Rwanda. Infect Agent Cancer 2014;9:40.

44 Koliopoulos G, Arbyn M, Martin-Hirsch P, et al. Diagnostic accuracy of human papillomavirus testing in primary cervical screening: a systematic review and metaanalysis of non-randomized studies. Gynecol Oncol 2007;104:232-46.

45 Castle PE, Smith KM, Davis TE, et al. Reliability of the Xpert HPV assay to detect highrisk human papillomavirus DNA in a colposcopy referral population. Am J Clin Pathol 2015;143:126-33.

46 Cuschieri K, Geraets D, Cuzick J, et al. Performance of a cartridge-based assay for detection of clinically significant human papillomavirus (HPV) infection: lessons from VALGENT (Validation of HPV genotyping tests). J Clin Microbiol 2016:54:2337-42. 